\title{
A Novel Small-World Immune Algorithm and It's Application to Robot Path Planning
}

\author{
Ye Du*, Kaiming Li, Xiong Xiao \\ School of Mechanical Engineering, Nanjing University of Science and Technology, China. \\ *Corresponding author. Tel.: 18251968272; email: dybetula@163.com \\ Manuscript submitted October 10, 2017; accepted January 8, 2018. \\ doi: $10.17706 /$ jcp.13.8.924-935
}

\begin{abstract}
Inspired by the mechanism of small-world phenomenon and immune-genetic algorithm, a novel algorithm is presented in this paper. By introducing the long-range operator and short-range operator in the small-world effect, the individuals with smaller fitness values after crossover are searched locally, and the individuals with high density and large fitness values are searched globally. Compared with the genetic algorithm and immune-genetic algorithm, results of function optimization show that the proposed algorithm has obviously improved the optimization capacity, efficiency and stability. Additionally, the algorithm is applied to the path planning problem of mobile robot. According to the density of obstacles in environment, a new adaptive division method is designed. Simulation results in multiple environments indicate that the new algorithm is characterized by improved search speed and short planning path, which verifies the validity of adaptive division and optimization performance of the algorithm.
\end{abstract}

Key words: Adaptive division, immune-genetic algorithm, mobile robot, path planning, small-world effect.

\section{Introduction}

The small-world phenomenon-the principle that any two individuals in the network are connected through a short sequence of intermediate acquaintances-was presented by social psychologist Milgram in 1967 [1], also popularly known as "six degrees of separation". It has led to two discoveries. One was that short chains really existed, and the other was that people in society were able to find these chains without knowing much about the target individual [2]. In 1998, Watts and Strogatz established the WS model based on the characteristics of the small-world network [3]. Since then, the small-world phenomenon has gradually been valued and become a hotspot in artificial intelligence, complex system, and complexity theory. Inspired by the mechanism of small-world effect, some scholars have done research. Chen [4] pointed out that adjusting the different propagation probability parameters would affect the search characteristic of small-world. In 2006, Du et al. [5] first applied the small-world network to the optimization problem and proposed a small-world algorithm, which consisted of a local short-range searching operator and a random long-rage searching operator. By performing a local search in a neighborhood and a global search in a non-neighborhood, it is effective to avoid trapping into the local minimum. Yuan et al. [6] took the individual density, constructed according to the information entropy, and fitness as the evaluation criterion for individual replacement, and the individual local search is performed by chaos disturbance after local short-range search, thus the searching capacity and efficiency are heightened. 
Path planning is an important issue in mobile robotics. In general, the problem can be described as searching an optimal and collision-free path from the starting point to the goal in an environment with obstacles [7]. According to the working environment of robot, the model of path planning can be divided into two types: global and local. In case of the global path planning, the environment is precisely defined and the planning is based on the given information. As for the local path planning, the environment information is unknown or partially unknown and the size, shape, and location of obstacles must be detected by sensors. According to the stage of method, and whether the method itself has the self-learning and self-organization ability, it can also be divided into two categories: traditional methods and intelligent methods. The former mainly includes free space approach [8], visibility graph method [9], [10], artificial potential field method [11], [12] and so on. In recent years, with the development of artificial intelligence, intelligent planning has aroused widespread concern of scholars both at home and abroad, including genetic algorithm (GA) [13], immune algorithm (IA) [14], ant colony algorithm (ACA) [15] and other planning methods. A GA has a global search capacity but it searches slow, whereas IA use learning, memory, and associative retrieval to solve recognition relevant patterns and remember patterns, which improve the premature convergence and slow convergence of GA [16], [17]. Yun et al. [18] combines GA with grid method, but the division of two-dimensional grid has a great influence on the result of path planning. Compared with the two-dimensional encoding, the computational complexity of the existing one-dimensional encoding is smaller. However, the problem of thickness of the division based on grid model still exists in one-dimensional model due to the equal division, and the planning is limited to the density of the division.

In this paper, a novel small-world immune algorithm (SWIA) is proposed. To improve the search ability and efficiency, the long-range operator and short-range operator in the small-world effect are introduced into the immune-genetic algorithm, and the novel algorithm is applied to robot path planning.

\section{SWIA Based on Small-World and Immune-Genetic Algorithm}

\subsection{Description and Definition of Small-World Optimization}

The small-world phenomenon revealed the most effective way of information transmission in many complex networks of objective world. The small-world network constructed by Watts and Strogatz is an intermediate network that extends from a regular network to a random network [2]. There are $n$ nodes distributed on a circle where each node is connected to its $k$ nearest neighbors and each node keeps a small number of links to some randomly chosen distant nodes with probability $p$. Links to neighboring nodes are called cluster links, whereas links to distant nodes are called long-links. Fig. 1 shows the principle of the small-world network when $n=12, k=4$. As $p$ increases, the network becomes increasingly disordered.

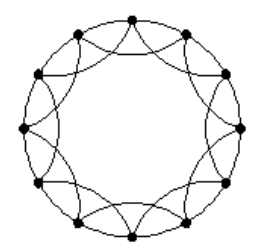

(a) $p=0$

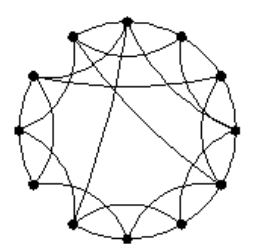

(b) $p=0.15$

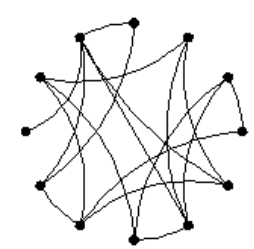

(c) $p=1$

Fig. 1. Principle of the small-world network.

As for the optimization problem based on small-world, the optimization can be regarded as a process where the solution of the optimal model transmits from the candidate node to the optimal model while small-world effect is considered during the search. 
Without loss of generality, the following optimization model is considered.

$$
y=\min (f(x)) \quad\left(x \in \boldsymbol{R}^{n}\right)
$$

where $x=\left[x_{1}, x_{2}, \cdots, x_{n}\right]^{T}, n$ is the dimension of $\boldsymbol{x}, f(\boldsymbol{x})$ is objective function.

To simplify the description of the proposed algorithm, some definitions are given as follows.

Definition $1 \boldsymbol{S}$ is the set of nodes (population) and $s$ represents a transfer node (individual), $s \in S . s$ is the binary encoding of vector $\boldsymbol{x}$, whereas $e^{-1}$ is written as a binary decoding mode, namely $x=e^{-1}(s)$. The total string length of $s$ is $M$.

Definition 2. For $\forall s_{i} \in S$, the $\lambda$ neighborhood of node $s_{i}$ can be defined as:

$$
\delta\left(s_{i}\right)=\left\{s_{j} \mid 0<\left\|s_{i}-s_{j}\right\| \leq \lambda, s_{j} \in \boldsymbol{S}\right\}
$$

where $\left\|s_{i}-s_{j}\right\|$ is the Hamming distance.

Definition 3. For $\forall s_{i} \in \boldsymbol{S}$, the non- $\lambda$ neighborhood of node $s_{i}$ can be defined as:

$$
\overline{\delta\left(s_{i}\right)}=\left\{s_{j}\left\|s_{i}-s_{j}\right\|>\lambda, s_{j} \in \boldsymbol{S}\right\}
$$

Definition 4. The average information entropy is defined as

$$
\begin{gathered}
H(N)=\frac{1}{M} \sum_{j=1}^{M} H_{j}(N) \\
H_{j}(N)=-\sum_{i=0}^{1} p_{i j} \log _{i j}
\end{gathered}
$$

where $H_{j}$ is the information entropy of the $j^{\text {th }}$ position of $N$ individuals, $p_{i j}$ is the probability that the $j^{t h}$ position of $N$ individuals is symbol $i$.

Definition 5. The similarity between individuals $s_{i}$ and $s_{j}$ is defined as

$$
A_{i j}=\frac{1}{1+H(2)}
$$

Definition 6. The concentration of individuals $s_{i}$ is defined as

$$
\begin{gathered}
C_{i}=\frac{1}{N} \sum_{j=1}^{N} Q_{i j} \\
Q_{i j}= \begin{cases}1 & A_{i j}>\text { Tacl } \\
0 & \text { otherwise }\end{cases}
\end{gathered}
$$

where Tacl is a preset value.

\subsection{Small-World Immune Algorithm Operators}

The small-world optimization algorithm mainly includes a local short-range searching operator $\boldsymbol{X}$ and a random long-range searching operator $\boldsymbol{Y}$. Let $\delta\left(s_{i}(k)\right)$ be the local search space of node $s_{i}(k)$, the main action of operator $\boldsymbol{X}$ is to transmit information to node $s_{i}(k+1)$ which is the nearest node from the target solution, that is

$$
s_{i}(k+1) \leftarrow \boldsymbol{X}\left(s_{i}(k)\right)=\left\{s_{i}^{\prime}(k) \mid f\left(e^{-1}\left(s_{i}^{\prime}(k)\right)\right)=\min \left(f\left(e^{-1}\left(s_{j}(k)\right)\right)\right), s_{j}(k) \in \delta\left(s_{i}(k)\right)\right\}
$$

Given the preset long-range probability $P_{l}$ and the non- $\lambda$ neighborhood $\overline{\delta\left(s_{i}(k)\right)}$, the operator $\boldsymbol{Y}$ randomly selects a node for the long-distance transmission of information, that is 


$$
s_{i}(k+1) \leftarrow \boldsymbol{Y}\left(s_{i}(k)\right)=\left\{s_{i}^{\prime \prime}(k) \mid s_{i}^{\prime \prime}(k) \in \overline{\delta\left(s_{i}(k)\right)}, P<P_{l}\right\}
$$

In order to simplify the flow of the SWIA, the selection, crossover, and mutation operators of immune-genetic algorithm are denoted as $\boldsymbol{I}_{s}, \boldsymbol{I}_{c}, \boldsymbol{I}_{m}$. In the method, the concentration is introduced to prevent the local convergence and improve the search efficiency. Before the select operation, calculate the concentration of individuals based on the information entropy. The higher the concentration is, the individual is inhibited more. And then the individuals are selected by the fitness and concentration. In addition, the immune-genetic algorithm has a memory bank that individuals with good information could retain to the next generation.

\subsection{Flow of the Small-World Immune Algorithm}

Step 1. Initialize the algorithm parameters: $n, m, l, P_{s}, P_{c}, P_{m}, P_{l}, k_{\max }$

Step 2. Generate the initial population $S(k)=\left[s_{1}(k), s_{2}(k), \cdots, s_{n}(k)\right]^{T}$ and calculate the fitness of the individuals.

Step 3. The individuals with smaller fitness values are extracted into the memory bank $s^{m}(k)$.

Step 4. Selection operator $\boldsymbol{I}_{s}$ is performed by roulette, that is

$$
\boldsymbol{S}^{\prime}(k) \leftarrow \boldsymbol{I}_{s}\left(\boldsymbol{S}(k) \mid P_{s}\right)=\left[s_{1}^{\prime}(k), s_{2}^{\prime}(k), \cdots, s_{n}^{\prime}(k)\right]^{T}
$$

Step 5. Randomly select the cross point for single-point crossover according to the probability $P_{c}$ and get a new population, namely

$$
\boldsymbol{S}^{\prime \prime}(k) \leftarrow \boldsymbol{I}_{c}\left(\boldsymbol{S}^{\prime}(k) \mid P_{c}\right)=\left[s_{1}^{\prime \prime}(k), s_{2}^{\prime \prime}(k), \cdots, s_{n}^{\prime \prime}(k)\right]^{T}
$$

Step 6. Assuming that the fitness value of $s_{i}^{\prime \prime}(k)$ is smaller than $s_{i}^{\prime \prime}(k+1)$ after the crossover operation, execute the local short-range operator $\boldsymbol{X}$ to $s_{i}^{\prime \prime}(k)$. If the fitness of $v_{i}^{\prime}(k)$ which is searched locally is smaller than $s_{i}^{\prime \prime}(k+1)$, then $v_{i}^{\prime}(k)$ is assigned to $s_{i}^{\prime \prime}(k+1)$.

$$
\begin{gathered}
6.1 \quad v_{i}(k) \leftarrow s_{i}^{\prime \prime}(k) . \\
6.2 \quad v_{i}^{\prime}(k) \leftarrow \boldsymbol{X}\left(v_{i}(k)\right) . \\
6.3 \quad \text { if } f\left(e^{-1}\left(v_{i}^{\prime}(k)\right)\right)<f\left(e^{-1}\left(s_{i}^{\prime \prime}(k+1)\right)\right) \text {, then } s_{i}^{\prime \prime}(k+1) \leftarrow v_{i}^{\prime}(k) .
\end{gathered}
$$

where $f\left(e^{-1}\left(s_{i}^{\prime \prime}(k+1)\right)\right)$ is the fitness value of $s_{i}^{\prime \prime}(k+1)$.

Step 7. According to the mutation probability $P_{m}$, the mutant individuals are bit-reversed to ensure the diversity of the population.

$$
\boldsymbol{S}^{\prime \prime \prime}(k) \leftarrow \boldsymbol{I}_{m}\left(\boldsymbol{S}^{\prime \prime}(k) \mid P_{m}\right)=\left[s_{1}^{\prime \prime \prime}(k), s_{2}^{\prime \prime \prime}(k), \cdots, s_{n}^{\prime \prime \prime}(k)\right]^{T}
$$

Step 8. Calculate the concentration of individuals, and sort the individuals according to the concentration. Then the individuals with high density and large adaptability are selected and crossed with new individuals which are generated randomly.

8.1 The sort operation $\boldsymbol{T}_{n}$ is executed according to the concentration, namely

$$
\boldsymbol{S}^{\prime \prime \prime \prime}(k) \leftarrow \boldsymbol{T}_{n}\left(\boldsymbol{S}^{\prime \prime \prime}(k)\right)=\left[s_{1}^{\prime \prime \prime}(k), s_{2}^{\prime \prime \prime}(k), \cdots, s_{n}^{\prime \prime \prime}(k)\right]^{T}
$$

For $\forall 1 \leq i \leq j \leq n, F\left(e^{-1}\left(s_{i}^{\prime \prime \prime}(k)\right)\right)>F\left(e^{-1}\left(s_{j}^{\prime \prime \prime}(k)\right)\right)$, where $F\left(e^{-1}\left(s_{i}^{\prime \prime \prime \prime}(k)\right)\right)$ is the concentration of $s_{i}^{\prime \prime \prime \prime}(k)$.

8.2 Choose the first $l$ individuals of $S^{\prime \prime \prime \prime}(k)$ and judge whether the condition $\left|f\left(e^{-1}\left(s_{i}^{\prime \prime \prime}(k)\right)\right)-f b e s t\right|>\varepsilon$ is met, where $f\left(e^{-1}\left(s_{i}^{\prime \prime \prime}(k)\right)\right)$ is the fitness value of $s_{i}^{\prime \prime \prime}(k)$, and fbest is the optimal solution for the objective function. If the condition is met, the individuals are crossed with new individuals which are generated randomly with 
given $P_{l}$. The crossover operation is the same with step 5.

Step 9. Execute the sort operation $\boldsymbol{T}_{n}$ according to the fitness value, update the population with the last $n-m$ individuals and the memory bank.

$$
9.1 \quad \boldsymbol{S}^{\prime \prime \prime \prime \prime}(k) \leftarrow \boldsymbol{T}_{n}\left(\boldsymbol{S}^{\prime \prime \prime \prime \prime}(k)\right)=\left[s_{1}^{\prime \prime \prime \prime \prime}(k), s_{2}^{\prime \prime \prime \prime}(k), \cdots, s_{n}^{\prime \prime \prime \prime \prime}(k)\right]^{T} .
$$

For $\forall 1 \leq i \leq j \leq n, f\left(e^{-1}\left(s_{i}^{\prime \prime \prime \prime}(k)\right)\right)>f\left(e^{-1}\left(s_{j}^{\prime \prime \prime \prime}(k)\right)\right)$.

$$
9.2 S(k)=S^{0}(k) \cup S^{m}(k) .
$$

where $s^{0}(k)$ are the last $n-m$ individuals of $s^{\prime \prime \prime \prime '}(k)$.

Step 10. Asses whether the termination condition $k=k_{\max }$ is met. If not, go to step 3, otherwise output the optimal solution and end.

\subsection{Function Optimization and Tests}

To verify the optimization performance of the proposed small-world immune algorithm, the following six functions are provided: Camel function $f_{1}$, Goldstein-Price function $f_{2}$, Shekel's Foxholes function $f_{3}$, Rastrigrin function $f_{4}$, Bohachevsky function $f_{5}$, Ackley's Path function $f_{6}$. Tests are implemented on a computer by using Matlab 7.0, and the test results of SWIA are compared with those of the GA and the IGA.

1) Camel function $f_{1}$

$$
\operatorname{minf} f_{1}=\left(4-2.1 x_{1}^{2}+\frac{x_{1}^{4}}{3}\right) x_{1}^{2}+x_{1} x_{2}+\left(-4+4 x_{2}^{2}\right) x_{2}^{2} \quad x_{i} \in[-5.12,5.12] \quad 1 \leq i \leq 2
$$

2) Goldstein-Price function $f_{2}$

$$
\begin{aligned}
\operatorname{minf}_{2}=\left[1+\left(x_{1}+x_{2}+1\right)^{2} \bullet\right. & \left.\left(19-14 x_{1}+3 x_{1}^{2}-14 x_{2}+6 x_{1} x_{2}+3 x_{2}{ }^{2}\right)\right] \bullet[30+ \\
& \left.\left(2 x_{1}-3 x_{2}\right)^{2} \bullet\left(18-32 x_{1}+12 x_{1}^{2}+48 x_{2}-36 x_{1} x_{2}+27 x_{2}{ }^{2}\right)\right] \quad x_{i} \in[-2,2] \quad 1 \leq \leq 2
\end{aligned}
$$

3) Shekel's Foxholes function $f_{3}$

$$
\operatorname{minf}_{3}=\left\{\frac{1}{K}+\sum_{j=1}^{25} \frac{1}{c_{j}+\sum_{i=1}^{2}\left(x_{i}-a_{i j}\right)^{6}}\right\}^{-1} \quad x_{i} \in[-65.536,65.536] \quad 1 \leq \leq 2
$$

$c_{j}=j \quad K=500$

$\left(a_{i j}\right)=\left[\begin{array}{l}-32,-16,0,16,32,-32,-16,0,16,32,-32,-16,0,16,32,-32,-16,0,16,32,-32,-16,0,16,32 \\ -32,-32,-32,-32,-32,-16,-16,-16,-16,-16,16,16,16,16,16,32,32,32,32,32,0,0,0,0,0\end{array}\right]$

4) Rastrigrin function $f_{4}$

$$
\operatorname{minf}_{4}=20+x_{1}{ }^{2}-10 \cos \left(2 \pi x_{1}\right)+x_{2}{ }^{2}-10 \cos \left(2 \pi x_{2}\right) \quad x_{i} \in[-5.12,5.12] \quad 1 \leq \leq 2
$$

5) Bohachevsky function $f_{5}$

$$
\operatorname{minf} 5=x_{1}^{2}+x_{2}^{2}-0.3 \cos \left(3 \pi x_{1}\right)+0.3 \cos \left(4 \pi x_{2}\right)+0.3 \quad x_{i} \in[-1,1] \quad 1 \leq \leq 2
$$

6) Ackley's Path function $f_{6}$

$$
\operatorname{minf}_{6}=-20 \exp \left(-0.2 \operatorname{sqrt}\left(0.5\left(x_{1}{ }^{2}+x_{2}{ }^{2}\right)\right)\right)-\exp \left(0.5\left(\cos \left(2 \pi x_{1}\right)+\cos \left(2 \pi x_{2}\right)\right)\right)+\exp (1)+20 \quad x_{i} \in[-30,30] \quad 1 \unlhd \leq 2
$$

In the simulation, parameters are set as follows. The population size is taken as 20 , memory library is 5 , 
the selection, crossover, mutation probability are taken as $0.08,0.3,0.1$, respectively, and the random long-range probability is 0.6 . The maximum search generation is 1000 . Considering the randomness of the algorithm, 50 random tests are performed independently for each function optimization, and the termination condition is the same.

Table 1 shows the comparison results among the three algorithms. $N_{\text {best }}$ indicates the number of the optimal solution. $N_{\max }$ and $N_{\text {mean }}$ denote the maximum and average convergence generation. $N_{\text {std }}$ indicates the standard deviation. As can be seen, the global optimization ability of IGA is improved through the immune operation, but compared with SWIA, the advantage is not obvious. For the six-peak hump function $f_{1}$ and multi-peak function $f_{3}$, the search capacity of IGA is not ideal. Due to the adoption of long-range and short-range operators in small-world effect, the SWIA proposed in this paper is able to retain the diversity of the population, and the search process is not easy to fall into the local minimum. Thus, the ability to search the global optimal solution is improved, and SWIA is able to find the optimal solution in all the tests. From the performance of $N_{\text {best }}$ and $N_{\text {mean }}$, we can see the strong search ability and quick convergence speed of SWIA. $N_{\text {std }}$ indicates that the robustness and stability of SWIA are better than those of GA and IGA, and it's not easily affected by external conditions.

Table 1. Test Results of GA, IGA and SWIA

\begin{tabular}{|c|c|c|c|c|c|c|c|c|c|c|c|c|c|}
\hline \multirow{2}{*}{ Function } & \multirow{2}{*}{$\varepsilon$} & \multicolumn{3}{|c|}{$N_{\text {best }}$} & \multicolumn{3}{|c|}{$N_{\max }$} & \multicolumn{3}{|c|}{$N_{\text {mean }}$} & \multicolumn{3}{|c|}{$N_{s t d}$} \\
\hline & & $\mathrm{GA}$ & IGA & SWIA & GA & IGA & SWIA & GA & IGA & SWIA & GA & IGA & SWIA \\
\hline$\overline{f_{1}}$ & $10^{-3}$ & 31 & 39 & 50 & 953 & 867 & 423 & 302.29 & 150.31 & 101.84 & 242.24 & 185.91 & 98.63 \\
\hline$f_{2}$ & $10^{-2}$ & 50 & 50 & 50 & 66 & 58 & 33 & 29.88 & 21.20 & 15.78 & 13.28 & 9.74 & 5.91 \\
\hline$f_{3}$ & $10^{-3}$ & 16 & 16 & 50 & 942 & 760 & 394 & 186.56 & 115.13 & 92.98 & 297.86 & 244.13 & 89.66 \\
\hline$f_{4}$ & $10^{-2}$ & 50 & 50 & 50 & 187 & 204 & 101 & 52.80 & 53.86 & 34.18 & 40.78 & 41.43 & 22.88 \\
\hline$f_{5}$ & $10^{-3}$ & 47 & 50 & 50 & 749 & 551 & 125 & 267.28 & 83.16 & 34.34 & 229.23 & 93.51 & 28.85 \\
\hline$f_{6}$ & $10^{-3}$ & 50 & 50 & 50 & 194 & 144 & 104 & 82.06 & 77.00 & 56.54 & 31.42 & 22.87 & 20.60 \\
\hline
\end{tabular}

Fig. 2 presents the test curves of function $f_{6}$. The known optimal solution of function $f_{6}$ is 0 . It can be noted that whether the evolutionary curves are for optimal solution or for the whole population, the function values obtained by the three algorithms are close to each other, but the convergence speed and average search capacity of SWIA are better than those of GA and IGA, which reflects that SWIA is superior to that of the other algorithms.

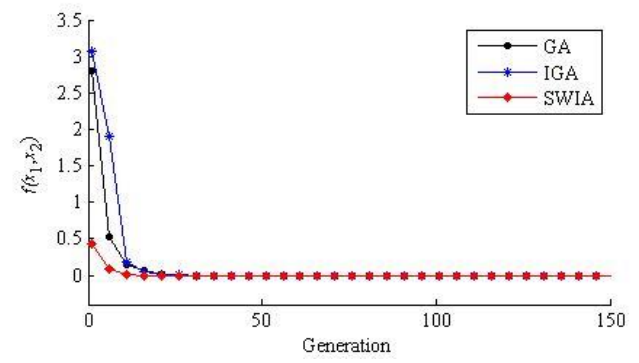

(a)Optimal solution

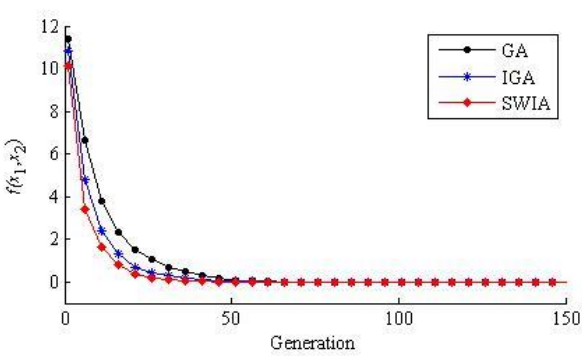

(b)Whole population

Fig. 2. Average evolutionary curves of function $f_{6}$.

\section{Path Planning with Small-World Immune Algorithm}

\subsection{Representation of Environment}

Many path planning methods use a grid-based model to represent the environment space. It has been determined that the planning effect is directly affected by the division of grid [19]. So a new adaptive 
division method is proposed in this paper. As shown in Fig. 3, there is a rectangular workspace of $8 * 6 \mathrm{~m}^{2}$ and different obstacles are set in space. $S$ and $E$ denote the starting point and the goal respectively. The blank area stands for free space where robot can move freely and the shadow stands for obstacle area. Considering the radius of the robot, the boundaries of the obstacles are expanded to a certain extent so that the robot can be treated as a point in the environment. Moreover, the height of robot is not considered in the two-dimensional space.

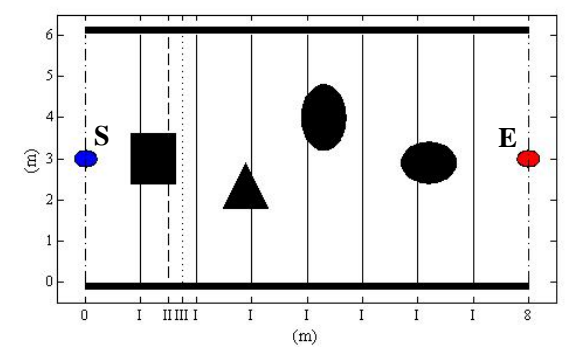

Fig. 3. Method of adaptive division.

As for current one-dimensional coding, the line between the starting location and target location is the $x$-axis of coordinate system, and then $x$-axis is divided into $n$ segments, so vertical $y$ can be used to express the code [20]. But the presented method is that $x$-axis is divided according to the density of obstacles. When the obstacles are almost full of space or less distributed in space, the space is not divided. The division consists of the following steps.

Step 1. Draw a line between $S$ and $E$ and divide it into $n$ segments. Vertical lines are drawn at the dividing points, denoted as division $I$. Let $\left[A_{1}, A_{2}, \cdots, A_{i}, \cdots, A_{n}\right]$ be the rectangular spaces. The following division orders are recorded as II, III, and so on.

Step 2. Calculate the length which the obstacles are crossed with space $A_{i}$ and denote it as $B$. If the condition $t_{1}<B<t_{2}$ is met, go to step 3 , otherwise step 4 , where $t_{1}$ and $t_{2}$ are conditions to determine the density of obstacles.

Step 3. $A_{i}$ is divided into $A_{i}$ and $A_{i+1}$. For space whose subscript is larger than $i$, subscript is incremented by 1 , return to step 2 .

Step 4. If $i$ is the largest subscript of the current space, the division is terminated, otherwise execute the operation: $i=i+1$ and go to step 2 .

\subsection{Initialization of Population}

The initial population is generally generated randomly. After the adaptive division of environment, a series of points are obtained. Therefore, a potential path is formed by line segments connecting the points generated on vertical lines (including $S$ and $E$ ). Each path must be checked whether it intersects an obstacle. If it does, the intersected point is changed randomly with a feasible one.

\subsection{Fitness Function}

The purpose of robot path planning is to find an optimal path between the starting point to the goal. Optimal path may be the shortest, the least time or the minimal working cost. The fitness function is used to evaluate the solutions. In this paper, the length of the planned path is taken as the objective function. So the individual with the smallest fitness value is the optimal path in current generation.

\subsection{Operators for Path Planning}

(1) Selection method

At this stage, objective function values of all individuals are obtained and assigned to them. Individuals 
with small fitness values will be selected with high probability which will accelerate the efficiency of planning, whereas individuals with large values has possibility to be selected which guarantee the diversity.

(2) Crossover operator

Two individuals are randomly selected, and the segments that can be exchanged are recorded. Then extract the segment and swap the points of the two paths after the segment. The new paths do not intersect an obstacle.

(3) Short-range operator

Let $L_{1}$ and $L_{2}$ be the two paths after crossover operation, and assume that the length of $L_{1}$ is shorter. A new path is formed with new points that are generated near the randomly selected points on $L_{1}$, denoted as $L_{3}$. If the length of $L_{3}$ is shorter than $L_{2}$, then $L_{3}$ is assigned to $L_{2}$.

(4) Mutation operator

This operation selects one mutation point randomly, and then a line segment connecting the points before and after the mutation point is formed. If the segment is infeasible, the position of the mutation point is adjusted on the vertical line. The criterion of successful mutation is that there is no collision and distance becomes shorter.

(5) Long-range operator

Calculate the concentration of paths after mutation and sort them according to the concentration. The first $l$ individuals with high density are chosen and determined. If the fitness value is quite different from the best individual in the current generation, crossover operation is performed between the individual and a new generated individual.

\subsection{Planning Simulations}

In order to demonstrate the effectiveness of SWIA in path planning, it is applied to four different environments and compared with the GA and IGA. The simulation experiments are carried out on MATLAB. As in Fig. 4, environment 1 and 2 are relatively simple, whereas environment 3 and 4 are complicated and there are obstacles before the target. The parameters are the same as the function optimization. For the three algorithms, there are two division methods: equal division and adaptive division. Considering the randomness, each intelligent algorithm in each environment is conducted 30 times for each method. The maximum generation is taken as 300 .

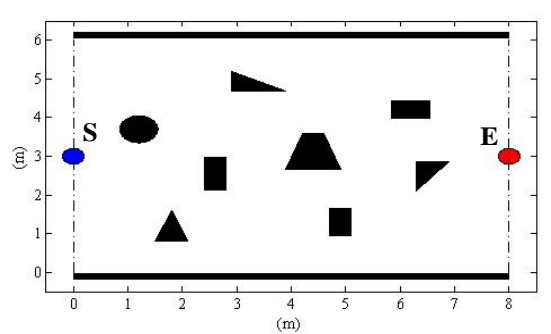

(a) Environment 1

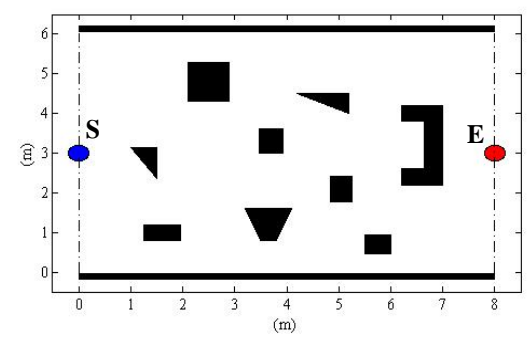

(c) Environment 3

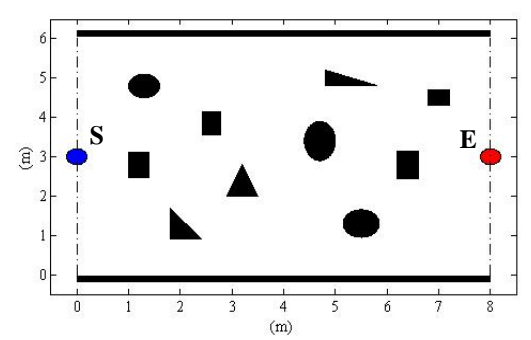

(b) Environment 2

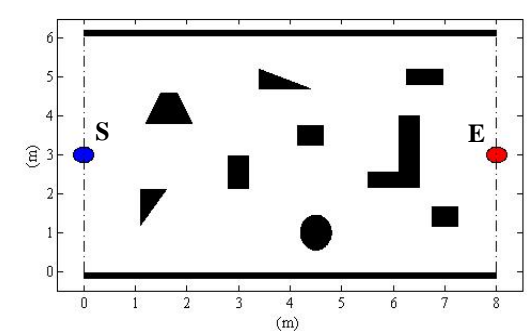

(d) Environment 4

Fig. 4. Four environments for path planning. 
Table 2 gives the experimental results for the four environments. Compared with equal division, it is clearly seen that the proposed method of adaptive division has obviously improved the search efficiency and the capacity to search for the shortest path. For the same division method, the three algorithms are able to converge to the optimal and collision-free path. Due to the local search of shorter path and the global search of longer path, the optimal path found by SWIA is the shortest which reflects the advantage of the presented algorithm in path planning. From the average length of all paths in the 30 experiments, we can see that the results of the SWIA are better than those of GA and IGA as well. The average convergence generation shows that the convergence speed of SWIA is the fastest which fully illustrates the search efficiency of SWIA.

Table 2. Planning Results among the Three Algorithms

\begin{tabular}{ccccccccccc}
\hline \multirow{2}{*}{ Environment } & \multicolumn{3}{c}{ Optimal path length } & \multicolumn{3}{c}{ Average path length } & \multicolumn{3}{c}{ Average convergence generation } \\
\cline { 3 - 11 } & & GA & IGA & SWIA & GA & IGA & SWIA & GA & IGA & SWIA \\
\hline \multirow{2}{*}{1} & Equal division & 8.3238 & 8.3883 & 8.1390 & 10.1034 & 10.1692 & 9.8980 & 184.80 & 225.47 & 167.37 \\
\cline { 2 - 12 } & Adaptive division & 8.1320 & 8.1395 & 8.1009 & 9.6957 & 9.6347 & 9.3458 & 127.70 & 138.40 & 113.60 \\
\hline \multirow{2}{*}{2} & Equal division & 8.6100 & 8.4544 & 8.1887 & 10.9886 & 10.7057 & 10.3675 & 179.53 & 195.97 & 158.90 \\
\cline { 2 - 12 } & Adaptive division & 8.3389 & 8.2410 & 8.0627 & 10.3766 & 10.0175 & 9.4890 & 126.80 & 132.13 & 116.73 \\
\hline \multirow{2}{*}{3} & Equal division & 8.6134 & 8.6109 & 8.4448 & 10.8919 & 10.6067 & 10.4294 & 187.67 & 222.27 & 175.47 \\
\cline { 2 - 12 } & Adaptive division & 8.5163 & 8.5064 & 8.4399 & 10.0138 & 9.7438 & 9.2302 & 104.00 & 110.13 & 90.40 \\
\hline \multirow{2}{*}{4} & Equal division & 9.0693 & 8.6662 & 8.4429 & 11.1080 & 10.7548 & 10.0135 & 184.20 & 198.10 & 165.80 \\
\cline { 2 - 12 } & Adaptive division & 8.4479 & 8.4405 & 8.3831 & 10.0683 & 10.5362 & 9.6556 & 114.13 & 123.00 & 101.03 \\
\hline
\end{tabular}

Fig. 5 and Fig. 6 show the optimal planning results of the three algorithms in environment 2 and 4, respectively. Fig. 5 and Fig. 6 are the optimal paths under the circumstance of equal division, whereas Fig. 5 $\mathrm{b}$ and Fig. $6 \mathrm{~b}$ are the optimal paths after adaptive division. It can be noted that the paths searched by SWIA are the global optimal paths.

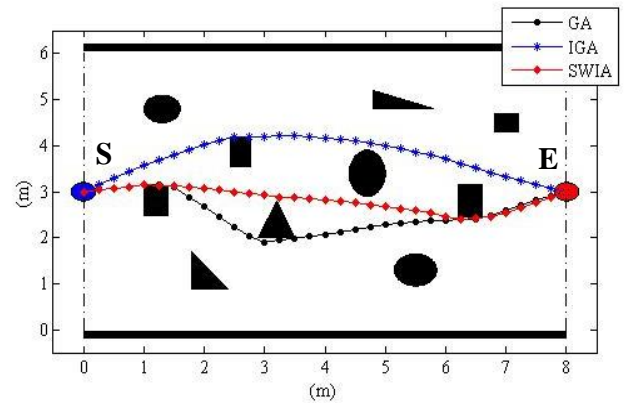

(a) Equal division

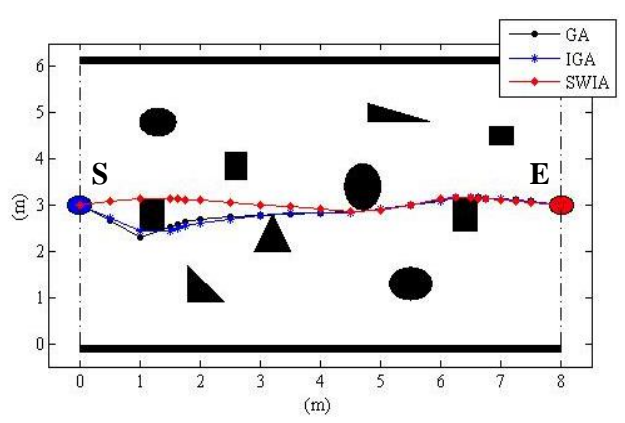

(b) Adaptive division

Fig. 5. Optimal planning results in environment 2.

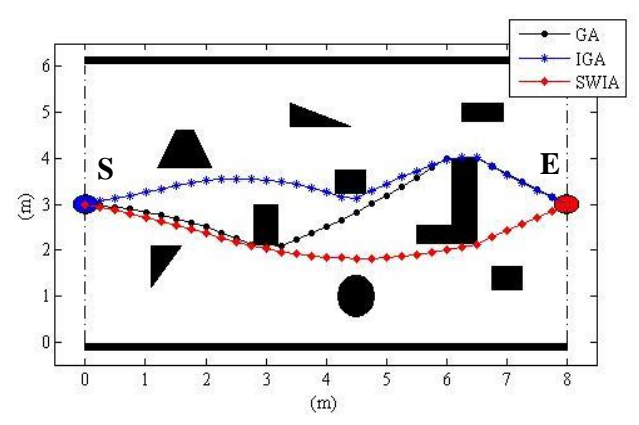

(a) Equal division

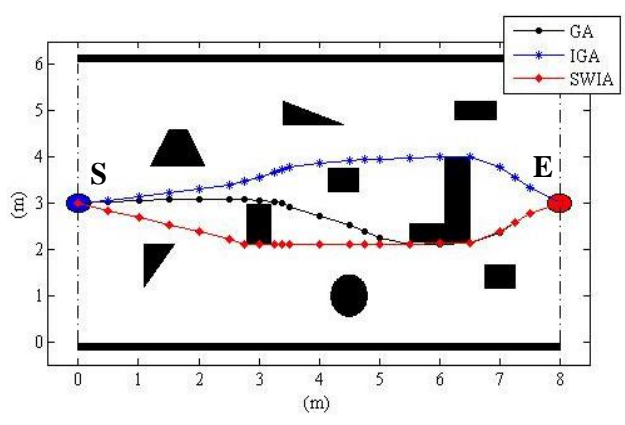

(b) Adaptive division

Fig. 6. Optimal planning results in environment 4. 
Fig. 7 and Fig. 8 illustrate the average convergence curves of the three algorithms in environment 2 and 4. From the two figures, we can see that the proposed SWIA converges more rapidly than the other algorithms do whether the average evolutionary curves are for equal division or for adaptive division.

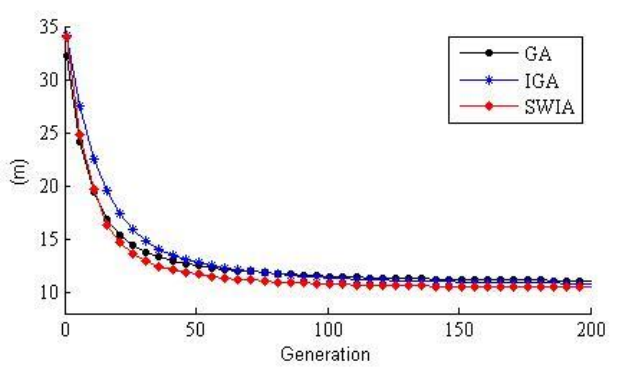

(a) Equal division

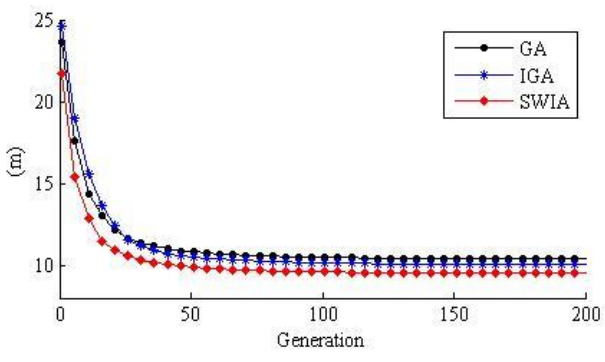

(b) Adaptive division

Fig. 7. Average convergence curves in environment 2.

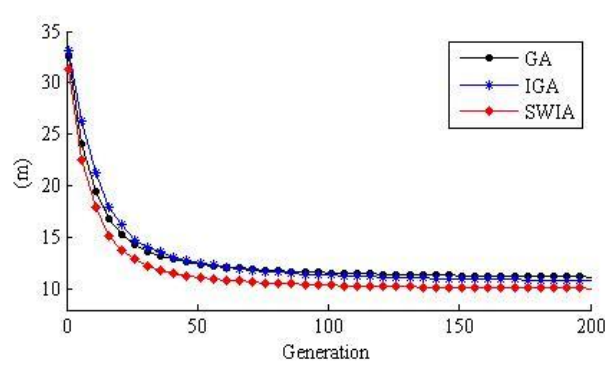

(a) Equal division

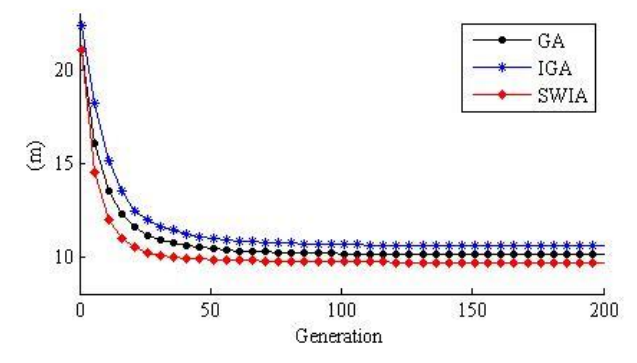

(b) Adaptive division

Fig. 8. Average convergence curves in environment 4.

\section{Conclusion}

In this paper, a novel algorithm(SWIA) is proposed on the basis of the small-world effect and immune-genetic algorithm. The local short-range searching operator and random long-range searching operator are introduced into the simple immune-genetic algorithm. It is clearly seen from the results of function optimization that SWIA can find the optimal solution for the most times and it converges more rapidly than the other methods do. Additionally, the novel algorithm is applied to solve the global path planning in complicated environments. According to the density of the obstacles, an adaptive division method is presented. The planning results among the three algorithms show that the proposed division method has obviously improved the search capacity and efficiency. For the same division method, the paths found by SWIA are shorter than those of GA and IGA and the convergence speed is the fastest, which demonstrate the effectiveness of SWIA in path planning. To further verify the validity of the proposed algorithm, experiments will be carried out in the following work, and the division method needs further discussion.

\section{Acknowledgment}

F. A. Author thanks Nanjing University of Science and Technology for the support of this work .

\section{References}

[1] Milgram, S. (1967). The small world problem. Psychology Today, 2(1), 185-195.

[2] Kleinberg, J. (2000). The small-world phenomenon: An algorithmic perspective. Proceedings of ACM Symposium on Theory of Computing, 406(2), 163-170.

[3] Watts, D., \& Strogatz, S. (1998). Collective dynamics of 'small-world' networks. Nature, 440-442. 
[4] Chen, J. Z., Liu, W., \& Zhu, J. Y. (2006). Two-dimensional small-world networks: Navigation with local information. Physical Review E Statistical Nonlinear \& Soft Matter Physics, 73(2), 056111.

[5] Du, H., Wu, X., \& Zhuang, J. (2006). Small-world optimization algorithm for function optimization. Advances in Natural Computation Pt, 4222, 264-273.

[6] Yuan, M., Du, H., Wang, S., \& Zhuang, J. (2008). Chaos small-world optimal algorithm based on population entropy. Journal of Xian Jiaotong University, 42(9), 1137-1141.

[7] Chen, X., Tan, G., \& Jiang, B. (2008). Real-time optimal path planning for mobile robots based on immune genetic algorithm. Journal of Central South University, 39(3), 577-583.

[8] Habib, M. K., \& Asama, H. (1991). Efficient method to generate collision free paths for an autonomous mobile robot based on new free space structuring approach. Proceedings of IEEE/RSJ International Workshop on Intelligent Robots and Systems '91. 'Intelligence for Mechanical Systems (pp. 563-567).

[9] Gao, B., Xu, D., Zhang, F., \& Yao, Y. (2009). Constructing visibility graph and planning optimal path for inspection of 2D workspace. Proceedings of IEEE International Conference on Intelligent Computing and Intelligent Systems (pp. 693-698).

[10] Yang, H., Xiao, X., \& Yao, D. (2009). A V-graph based global path planning algorithm for mobile robot. Journal of Shenyang University of Technology, 31(2), 225-229.

[11] Rimon, E. (1990). Exact robot navigation using artificial potential functions. IEEE Transactions on Robotics \& Automation, 8(5), 501-518.

[12] Shi, W., Huang, X., \& Zhou, W. (2010). Path planning of mobile robot based on improved artificial potential field. Journal of Computer Applications, 30(8), 2021-2023.

[13] Lu, J., \& Yang, D. (2007). Path planning based on double-layer genetic algorithm. Proceedings of International Conference on Natural Computation (pp. 357-361).

[14] Wang, Y. N., Hsu, H. H., \& Lin, C. C. (2005). Artificial immune algorithm based obstacle avoiding path planning of mobile robots. Proceedings of 1st International Conference on Advances in Natural Computation (pp. 859-862).

[15] Zong, Z., Jing, S., \& Jia, T. (2002). Application of the improved ant colony algorithm. Journal of Shanghai Jiaotong University.

[16] Dasgupta, D., \& Attoh-Okine, N. (1997). Immunity-based systems: A survey. Proceedings of IEEE International Conference on Systems, Man, and Cybernetics, Computational Cybernetics and Simulation (pp. 369-374).

[17] Dasgupta, D., \& Dasgupta, D. (1999). Artificial immune systems and their applications. Lecture Notes in Computer Science, 1(4), 121-124.

[18] Yun, S. C., Ganapathy, V., \& Chong, L. O. (2011). Improved genetic algorithms based optimum path planning for mobile robot. Proceedings of International Conference on Control Automation Robotics \& Vision (pp. 1565-1570).

[19] Tuncer, A., \& Yildirim, M. (2012). Dynamic path planning of mobile robots with improved genetic algorithm. Computers \& Electrical Engineering, 38(6), 1564-1572.

[20] Liu, L., Wang, Y. N., Kuang, F., \& Zhang, H. (2007). Path planning of mobile robot based on neural network and genetic algorithm. Application Research of Computers, 24(2), 264-261.

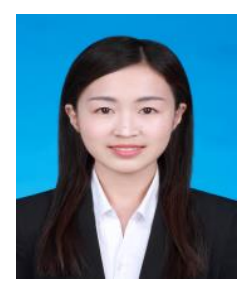

Ye Du was born in Hubei, China, in 1993. She was graduated from Jiangsu University of Science and Technology in 2015. Currently, She is a postgraduate student in the School of Mechanical Engineering at Nanjing University of Science and Technology, China. Her main research interest is intelligent detection and control. 


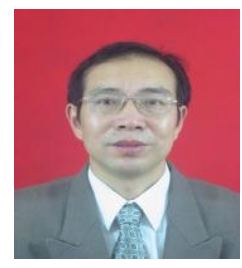

Kai Ming Li was born in Hubei, China, in 1961. He is a associate professor in the School of Mechanical Engineering at Nanjing University of Science and Technology, China. His main research subject is mechanical manufacturing and automation.

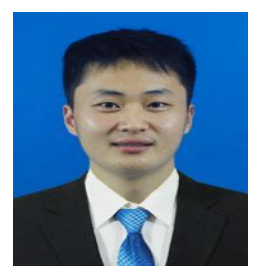

Xiong Xiao was born in Hubei, China, in 1993. He received his B.S degree in Nanjing University of Science and Technology in 2015. Currently, He continues to pursue a master's degree in this school. His research interest mainly focuses on control of mechatronic system. 